\title{
An advanced physiological data logger for medical imaging applications
}

\author{
Tareq Hasan Khan and Khan A Wahid ${ }^{*}$
}

\begin{abstract}
The interest of physiological data sensing and recording using wireless body sensor network has increased in recent years due to the advancement of miniature and portable electronic devices. In this study, the design of a portable and rechargeable data logger with high data rate multiple wireless connectivity (Bluetooth and 2.4-GHz radio frequency) is discussed. The data are logged in micro secure digital (SD) cards and can be transferred to PC or Smartphone using SD card reader, USB interface, or Bluetooth wireless link. Analog signals can also be logged through an 8-channel analog-to-digital interface. A graphical LCD with touch screen is added for control and diagnosis. The hardware is generic and targeted for various medical imaging and data collection applications. The functionality of the prototype is later tested for wireless capsule endoscopy and skin temperature logging application.
\end{abstract}

Keywords: Data logger, Bluetooth, Wireless link, Capsule endoscopy, Analog-to-digital

\section{Introduction}

Recent advances in miniature and portable bio-sensors, embedded processors, and wireless technologies have caused a rapid growth in sensing and recording physiological signals for medical applications. Bio-sensors convert body signals such as temperature, blood pressure, breathing [1], heartbeat [2-4], etc., to electrical signals to be processed, transmitted, and recorded in electronic devices [5]. Data coming from implanted or external body sensors can be transmitted wirelessly to a portable and wearable data logger unit, thus giving the patient the freedom to do household works during continuous data recording without the hassle of cumbersome wired devices. After logging is completed, the data are transferred to a personal computer (PC) or Smartphone which may be later examined by physicians. In this article, a portable, battery-operated data logger unit having wireless connectivity with the bio-sensor is discussed. The design of the data logger is general and can be used for several medical applications. In this article, the prototype is demonstrated for video wireless capsule endoscopy (WCE) [6] and skin temperature logging application.

\footnotetext{
* Correspondence: khan.wahid@usask.ca

Department of Electrical and Computer Engineering, University of Saskatchewan, Saskatoon, SK S7N5A9, Canada
}

Several works are found in the literature related to data logging for medical applications. In [7], a data logger unit for storing galvanic skin response for autistic patients is discussed. The data logger receives data using RS232 interface and stores it in a 16-MB EEPROM. In [2], the design of a data logger is discussed for electrocardiogram (ECG) signals. The design converts the ana$\log$ ECG signals to digital and stores them in 20-MB flash memory cards. An ECG signal data logger with custom-designed ASIC controller for multimedia card (MMC) is discussed in [3]. In [8], a fetal and maternal heart beat signal recorder is discussed which detects signals using skin electrodes, converts them to digital, and stores in temporary SRAM for later transfer to PC by RS232 interface. A portable data logger with three body-fixed inertial sensors for monitoring the physical activities of Parkinson's disease patients is proposed in [9]. In [10], the design of a microcontroller-based portable data logger for medical application is described which contains three-electrode ECG circuit, three accelerometers, a pressure sensor, and a temperature sensor. The module can store data in 4-Mb flash memory in real-time or can send data to PC by a wired serial interface. A low power and small size design of an ECG signal recorder is described in [11] for the purpose of long-term portable recording. Data are stored in secure 
digital (SD) card and can be transferred to $\mathrm{PC}$ by an isolated RS232 interface. In [12], fetal movements are recorded continuously on several pregnant patients using fetal movement acceleration measurement recorder to study its suitability in long-term home monitoring application. The work in [13] presents an IP core for FPGA-based ECG data transmission using wired telephone line through modem interface. Note that, the above-discussed data loggers receive data from body sensors using wired connections which is not comfortable for patients. In [14], a microcontroller-based data logger is implemented by using a four-channel analog-to -digital converter (ADC) to measure sweat activity. Data are stored in an EEPROM with the capability of storing data for over 2 days when one measurement is taken per minute. An RF transceiver is used to export the data to a monitoring host PC. A wireless data logger for recording human movements is discussed in [15]; however, the data logger is not portable as it takes power from house AC line supply, thus restricts the patient's movement near to the data logger. In [16], an ARM microcontroller-based wearable heart rate monitor system is described. It gathers ECE data and sends the data to a nearby PC wirelessly using Bluetooth. It does not have internal storage memory and thus the patient's movements are restricted near the host PC. In [17], a Java2-based software for cell phone is developed to store laboratory data, such as blood pressure, blood urea nitrogen, creatinine, $\mathrm{Hb}$ A1c (glycosylated hemoglobin), and other pertinent comments, into a cell phone memory. However, the cell phone does not have any sensor connected with it for automatic data acquisition.
The data logger presented in this study is portable and has high data rate of $2 \mathrm{Mbps}$ wireless connectivity with implantable or externally attached body sensors which eliminated the need for wires. It also has high memory capacity of $4 \mathrm{~GB}$ micro SD card, graphical display for showing images, graphs, charts in real time, keypad, and touch screen-based user interface. After logging, the data can be transferred to $\mathrm{PC}$ using an $\mathrm{SD}$ card reader at a speed of up to $25 \mathrm{MB} / \mathrm{s}$ or using an USB interface. Optionally, the data can also be transferred to $\mathrm{PC}$ or Smartphone wirelessly using Bluetooth technology. An illustration of a data logging system of a wireless body sensor system using the proposed data logger is shown in Figure 1.

\section{Design requirements}

In order to make an advanced data logger that is useful to various medical image and video application, we consider the following design requirements.

- The data logger must have both wired and wireless input connectivity so that it can be connected with various in-body sensors. For example, for accelerometer or heart monitoring system, it uses wired analog interface, whereas for applications like WCE, it uses wireless RF interface.

- The data logger must have high storage capacity for data intensive application. For example, in WCE, to store QVGA $(320 \times 240)$ color, 24 bits-per-pixel images transmitted at 2 frames-per-second (FPS) having $80 \%$ compression ratio (CR) for $10 \mathrm{~h}$, at least 3.1 GB memory space is required as calculated using (1).

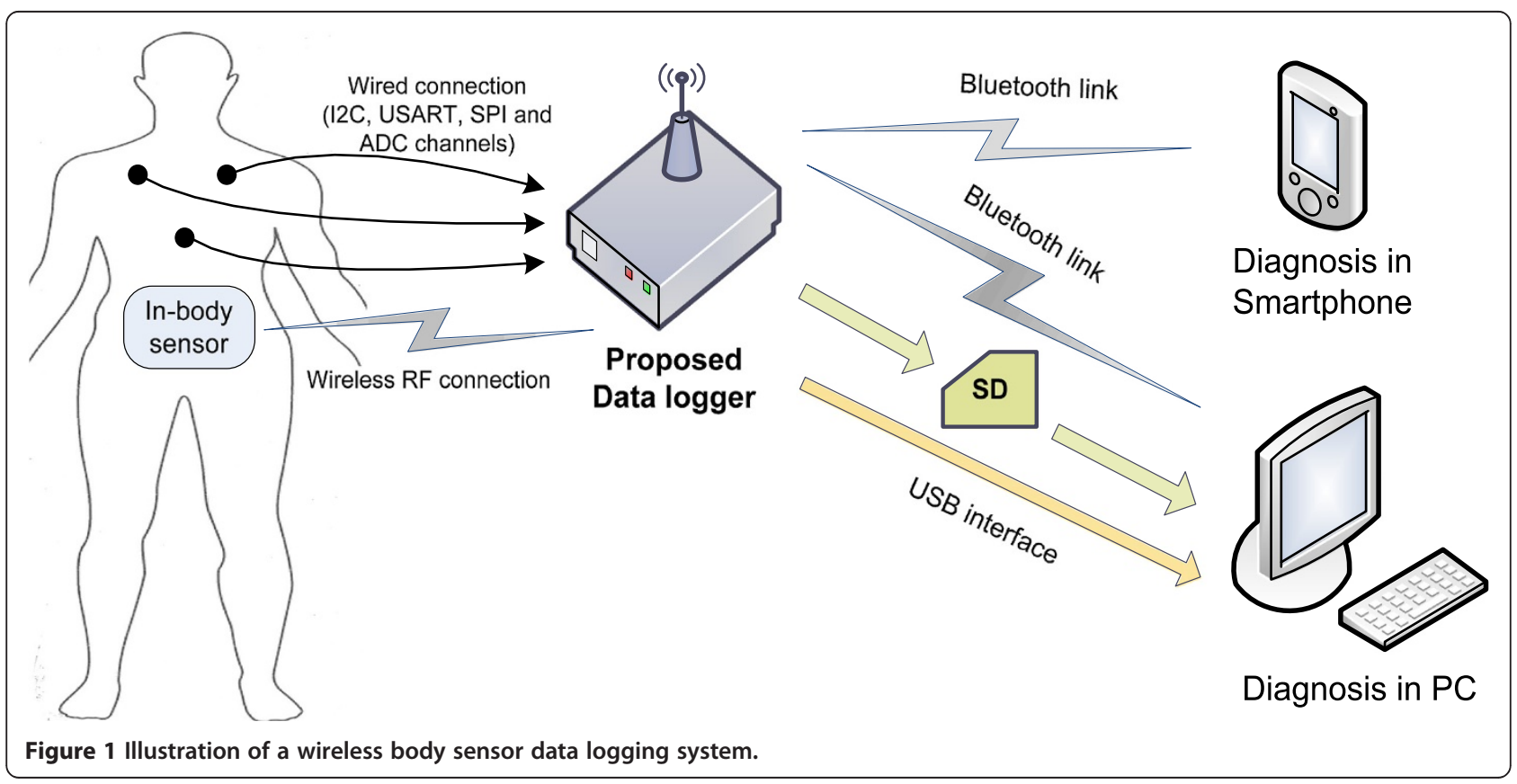




$$
\begin{aligned}
\text { Store capacity }= & \text { Width } \times \text { Height } \\
& \times \text { Bits Per Pixel } \times(1-C R) \\
& \times \text { FPS } \\
& \times \text { Logging time }(\text { in hour }) \\
& \times 3600
\end{aligned}
$$

- The logger must consume low power so that the battery runs for longer time. For example, in WCE, it must run continuously for at least $10 \mathrm{~h}$.

- The data rate of the wireless transceiver and the writing speed of the data logger must be high enough to support storing color video. For example, for QVGA resolution at 2 FPS and $80 \%$ CR, the required data rate of the wireless transceiver is at least $720 \mathrm{kbps}$. The writing speed in storage memory must be higher than $90 \mathrm{kB} / \mathrm{s}$ in this case.

- The data logger should be able to communicate with the bio-sensor in real-time during logging and control various features of the sensor, such as, changing image resolution, data rate, data types, etc. The interface used for this interactive control operation must be easy to use.

- Real-time viewing (RTV) of the data (i.e., images, charts, etc.) is another important feature. It will assist a physician performing diagnosis in real-time.

- After data logging is completed, there must be easy and fast way to transfer the data to workstation PC or Smartphone for diagnostics. As a result, we propose to have multiple wired and wireless output (or transfer) connectivity such as, USB interface, micro SD, and Bluetooth.

- To be wearable and easily portable, the physical size and weight of the data logger must be as less as possible.

\section{Methods}

The data logger is designed to have four layers as shown in Figure 2. Each layer completes its functionality by using the resources of its lower layers. By only changing the application layer firmware, the data logger can be used in several applications, without modifying the lower

\begin{tabular}{|c|}
\hline Application \\
\hline Disk Operating System \\
\hline Driver \\
\hline Hardware \\
\hline
\end{tabular}

Figure 2 Data logger design layers. level driver and hardware layers. A brief description of each layer is given in the following sections.

\section{Hardware}

The overall block diagram of the hardware is shown in Figure 3. The design consists of a microcontroller (MCU) and several peripherals connected with it. Each major component of the design is briefly described below.

\section{Microcontroller}

A microcontroller from the XMEGA-A1 [18] family is chosen for this design. A comparison of XMEGA with other microcontrollers is given in Table 1 . To increase the data sample rate and writing speed in the storage device, a high-speed MCU is required. Moreover, several peripherals are connected with the MCU using SPI and USART protocols. The XMEGA microcontroller has more hardware SPI and USART units than others which is an advantage. It has 78 programmable $\mathrm{I} / \mathrm{O}$ lines, 128$\mathrm{kB}$ flash for program storage, 8-kB SRAM, and 2-kB EEPROM to store user defined variables temporarily and permanently namely.

\section{Graphical $L C D$ with touch screen}

To enable RTV of images, graphs, charts, texts, etc., during data logging, a 2.4" graphical LCD [21] capable of displaying $320 \times 240$ pixels (QVGA) using 262,144 colors is chosen. The display also contains resistive touch area, enabling advanced and interactive user interface. The LCD connects with the MCU using hardware

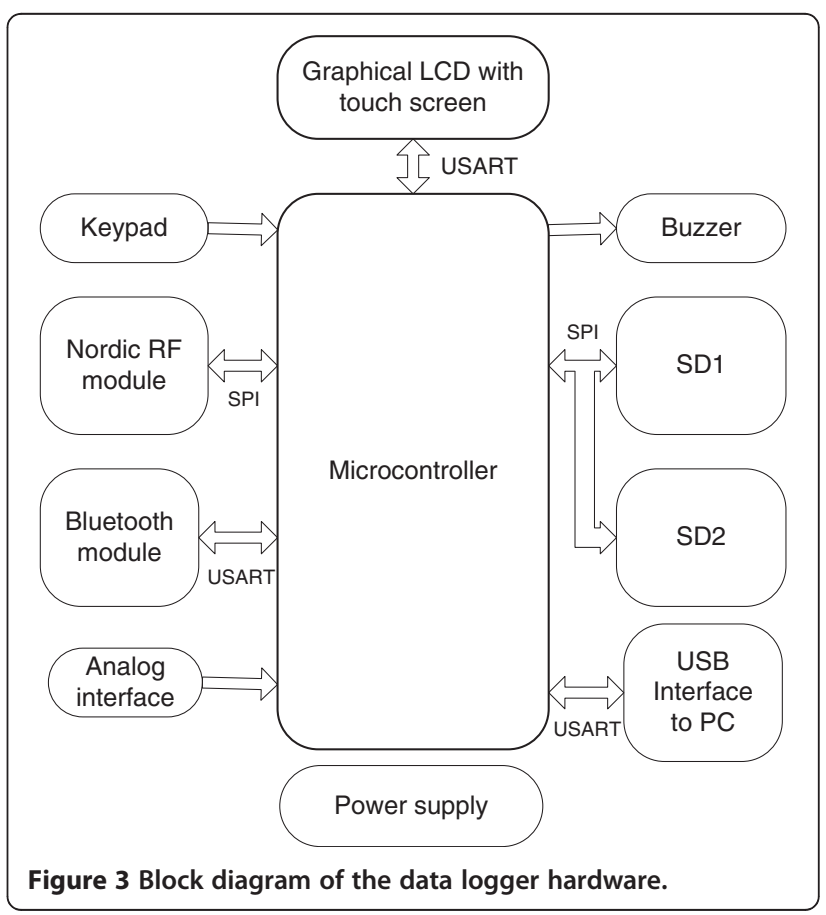


Table 1 Comparison of several MCU

\begin{tabular}{lcccccc}
\hline & Clock (MHz) & SRAM (kB) & Hardware SPI & Hardware USART & Core power (mW) & Unit price (USD) \\
\hline PIC (PIC16F1947) [19] & 32 & 1 & 2 & 2 & 11.8 & $\$ 3.20$ \\
ARM Cortex M3 (LPC1316FBD48) [20] & $25^{\text {a }}$ & 8 & 2 & 1 & 2 & 13.2 \\
AVR (ATMEGA644P) [18] & 20 & 4 & 1 & 8 & 87.5 & $\$ 7.50$ \\
XMEGA-A1 (ATXMEGA128A1) [18] & 32 & 8 & 4 & 86 & $\$ 10.20$ \\
\hline
\end{tabular}

acan be increased using PLL up to $72 \mathrm{MHz}$.

USART and it receives command from the MCU at a baud rate of 2 Mbps.

\section{Data storage device}

In Table 2, the comparisons of different permanent memory storage device in a single chip are shown [18]. Parallel EEPROMs need significant number of I/O lines to interface with MCU. Serial EEPROMs need relatively lesser I/O lines; however, their memory capacity is low and cascading several EEPROMs for increasing capacity, needs significant area, and more I/O lines. Micro SD card [22] seems to be the best choice as it has high memory capacity and needs only few I/O lines. Moreover, its defect and error management unit promises reliable data read/write. In the data logger design, two 2GB SD card are connected with the MCU using hardware SPI at a $16-\mathrm{MHz}$ clock speed. Note that, several SD cards may be connected as shown in Figure 4 and the storage capacity can be increased significantly. When one SD becomes full, data are written in another SD card automatically, and thus data logging can continue without the interruption of replacing SD card. Moreover, data can be transferred from SD card to $\mathrm{PC}$ using $\mathrm{SD} /$ MMC card reader at high speed such as $25 \mathrm{MB} / \mathrm{s}$.

\section{Wireless transceiver}

The characteristics of several wireless transceiver units are shown in Table 3. The major challenge of any wireless link is the data corruption in the transmission channel. Nordic transceiver [23] contains cyclic redundancy check (CRC)-based error detection and retry with auto acknowledgement (i.e., resend data packet until success)

Table 2 Comparison of different permanent memory storage device

\begin{tabular}{lccccc}
\hline & \multicolumn{4}{c}{ EEPROM } & Micro SD \\
\cline { 2 - 5 } & Parallel & \multicolumn{3}{c}{ Serial } & \\
\cline { 2 - 5 } & & I2C & SPI & $\mathbf{3}$ WIRE & \\
\hline Capacity & $512 \mathrm{kB}$ & $64 \mathrm{kB}$ & $64 \mathrm{kB}$ & $2 \mathrm{kB}$ & $2 \mathrm{~GB}^{\mathrm{a}}$ \\
Clock (MHz) & 5 & 0.4 & 20 & 2 & $25^{\mathrm{b}}$ \\
Pins required & 30 & 2 & 4 & 4 & 4 \\
Error correction & No & No & No & No & Yes \\
\hline
\end{tabular}

${ }^{a}$ Maximum capacity for FAT16 file-system for a single SD.

${ }^{\mathrm{b}}$ In standard SPI mode. feature which makes the link promisingly reliable, though several retry may decrease the overall data transmission rate in a noisy environment [15]. We selected Nordic for our application and it is connected with the MCU using hardware SPI at $8 \mathrm{MHz}$ speed.

\section{Bluetooth}

A Bluetooth transceiver [28] is also connected with the MCU using hardware USART at 230-kbps baud. Data transmitted from wireless body sensor can be captured either by Nordic or by Bluetooth in the data logger and then can be uploaded wirelessly to personal computer or Smartphone using Bluetooth.

\section{USB interface}

The design also contains an USB interface (as shown in Figure 5) that has 1-Mbps data rate to connect with PC using wired link. This wired interface can optionally be used to transfer data to workstation $\mathrm{PC}$ after data logging.

\section{Analog interface}

Analog data can also be taken in the data logger using the wired analog interface. The analog interface consists of multiplexed eight programmable ADC channels, eight analog comparators, and two digital-to-analog converter channels of the microcontroller. Analog data such as body temperature, blood pressure can be taken using the ADC channels and then the data can be saved in SD

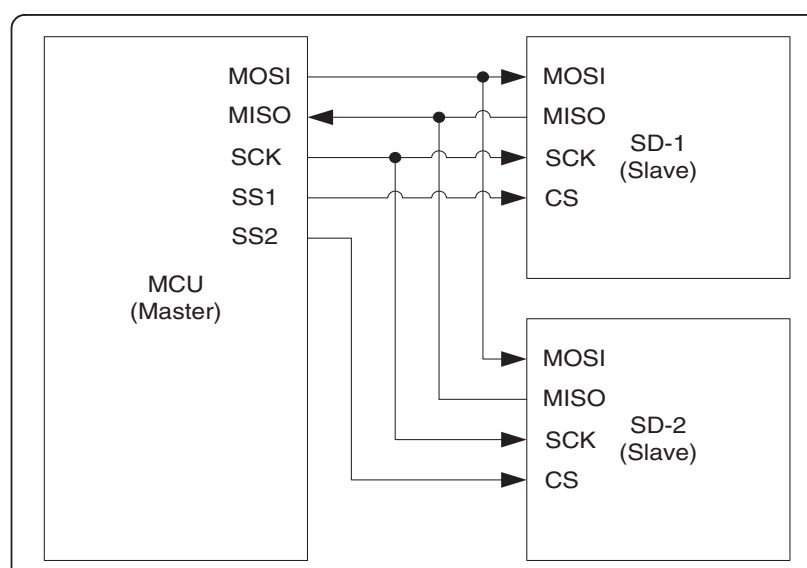

Figure 4 SD card connection with MCU by SPI. 
Table 3 Comparison of different wireless transceivers

\begin{tabular}{|c|c|c|c|c|c|}
\hline & Zarlink [24] & Linx [25] & Micrel [26] & Zigbee [27] & Nordic [23] \\
\hline Frequency band & $402 \mathrm{MHz}$ & $433 \mathrm{MHz}$ & $915 \mathrm{MHz}$ & $2.4 \mathrm{GHz}$ & $2.4 \mathrm{GHz}$ \\
\hline Data rate & $800 \mathrm{kbps}$ & $10 \mathrm{kbps}$ & 115 kbps & $250 \mathrm{kbps}$ & $2 \mathrm{Mbps}$ \\
\hline Retry and auto ack. & Yes & No & No & Yes & Yes \\
\hline TX current (mA) & 5 & 3.4 & 25 & 250 & 11.3 \\
\hline RX current (mA) & 5 & 5.2 & 13.5 & 55 & 13.5 \\
\hline
\end{tabular}

card and its real-time graphical plot can be displayed on LCD.

\section{Power supply}

As the power source of the data logger, three $3.7 \mathrm{~V}$ polymer lithium-ion battery, each having 2000-mAh rating are used in parallel, thus making total battery rating of 6000-mAh. The design also contains a 200-mA having 3.3-V output voltage boost converter [29] and an under voltage protection of $2.6 \mathrm{~V}$. A $500-\mathrm{mA}$ constant current battery charging circuit [30] using PC's USB port is also included in the design so that recharging can be done without removing the batteries from the hardware. To indicate and monitor the charging level, the battery voltage is sampled using a potentiometer and fed to an ADC channel of the microcontroller.

\section{Other peripherals}

To interact with the user, the design contains four push button switches. The button press is signaled to the MCU as interrupt. A buzzer is included in the design to generate small beep sounds.

\section{Driver firmware and disk operating system (DOS)}

The driver layer consists of low-level firmware for accessing different hardware peripherals. They are briefly described below.

\section{Graphical $L C D$ and touch screen}

The driver for graphical LCD contains functions for initializing the LCD, drawing pixels at any position by providing the co-ordinate and color information, setting background color and brightness, taking the LCD in low-power sleep and wake-up modes, etc. The touch screen driver functions provide the co-ordinate when a touch is made on the screen.

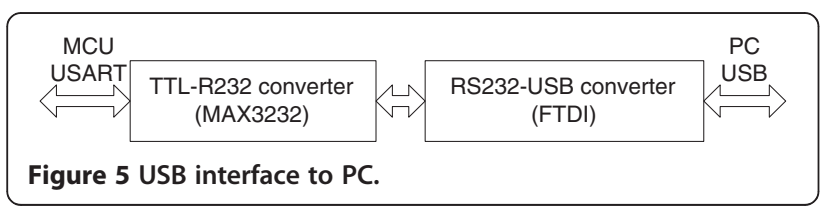

\section{Keypad}

When any of the four keys (Up, Down, Enter, and Escape) is pressed, the program jumps to its corresponding interrupt routine and sets a flag for that particular key. By executing a get_key () function, the last key pressed is detected by reading the flags.

\section{Wireless $R F$ transceiver}

It contains functions for reading and writing data to the configuration registers of Nordic RF module using SPI, setting the RF module as transmitter or receiver, flashing, and checking the status of internal Fast in, Fast out (FIFO) buffers, sending and receiving data packets to/from other RF module, taking the module to power down and up mode, etc.

\section{Bluetooth}

These functions initialize the Bluetooth device as slave, connect with nearby Bluetooth master devices (such as Smartphone or PC) when request is made, then send and receive data to/from master Bluetooth device using USART. It also contains functions to take the module in low-power sleep and wake-up modes.

\section{Buzzer}

To generate a "beep" sound, square pulses of $1 \mathrm{kHz}$ are sent for the duration of $500 \mathrm{~ms}$ to the buzzer.

\section{SD card and DOS}

It contains functions for checking whether any SD card is present in the slot, selecting and initializing the SD card, checking the available memory space, etc. A DOS for embedded system [31] is implemented which handles file operations. Using DOS, data files can be created, read, written, and deleted. The DOS implements FAT16 file system and the maximum writing speed in SD card is found to be $115 \mathrm{kB} / \mathrm{s}$ when the MCU is running at 32 $\mathrm{MHz}$.

\section{USB interface}

This driver sends and receives data to/from PC. Data are transferred from the MCU using USART protocol and through an RS232-USB converter chip, data are packed according to USB protocol to transmit to PC through USB port. 


\section{Analog interface}

It configures the ADC resolutions (such as 8, 10, 12 bit), reference voltage, channel gain, etc. It provides functions using which the corresponding digital data of an analog channel can be read and processed. It also contains functions to check battery voltages in the Power Supply module.

\section{Application firmware}

This layer contains the firmware designed for a specific data logging application. The proposed data logger is generic that encompasses many different medical applications such as ECG, heartbeat, capsule endoscopy, blood pressure, etc. In this study, we have implemented the firmware for capsule endoscopy application [6].

\section{User interface}

In order to set different settings such as image size, imaging mode, enable/disable real-time view, etc., a menubased graphical user interface is designed in the data logger as shown in Figure 6. User can use either four keys (Up, Down, Enter, and Escape) or touch to select different menu options. Additional features may easily be added to the interface if needed.

\section{Data logging}

In order to receive data from the bio-sensor (i.e., capsule in this case study), a command is sent wirelessly to the capsule from the data logger containing information of required image size and imaging mode. After receiving command, the capsule starts to send the data packets of an image frame in compressed from. The data logger reads the packets and stores them in SD card. A timer is also used to add time stamp for each image frame. The end of a frame is detected by a pattern of consecutive four zero bytes. The overall data logging procedure is shown in Figure 7.

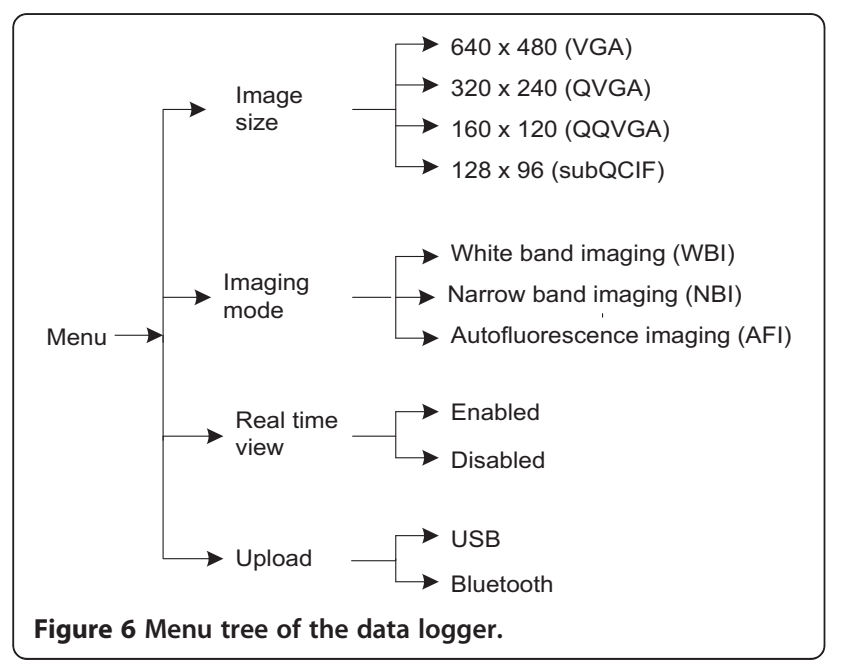

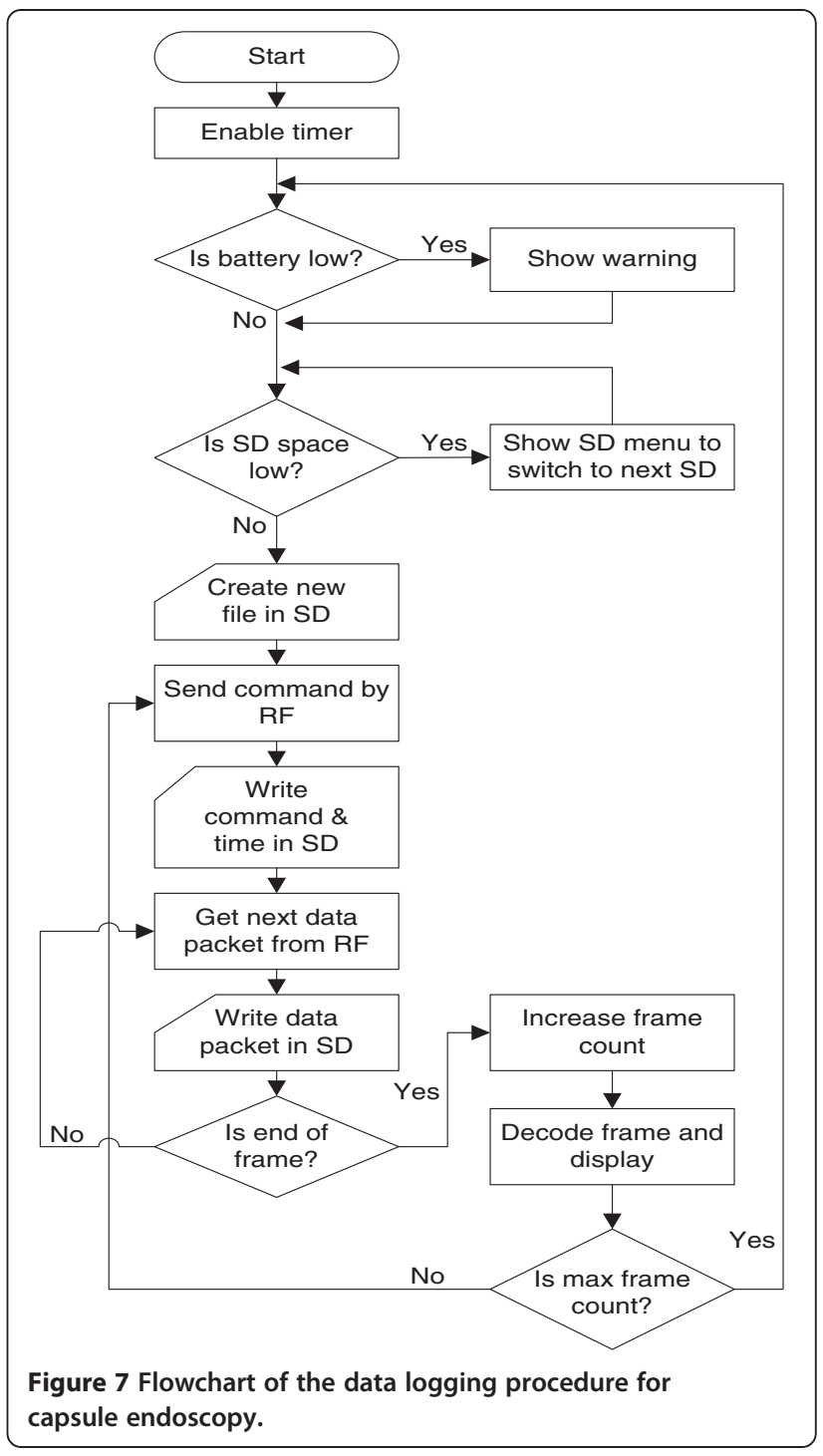

\section{Image decoding algorithm}

In capsule endoscopy, image data are transmitted wirelessly from the ingested capsule in compressed form. Our proposed compression algorithm consists of a novel color space, YEF [32], which is designed by analyzing the unique properties of endoscopic images for better compression. After converting RGB pixels to YEF color space, the compressor takes the difference of consecutive pixels (left pixel prediction) and then encodes the differences in variable length coding such as in Golombrice code. Based on the nature of endoscopic images,

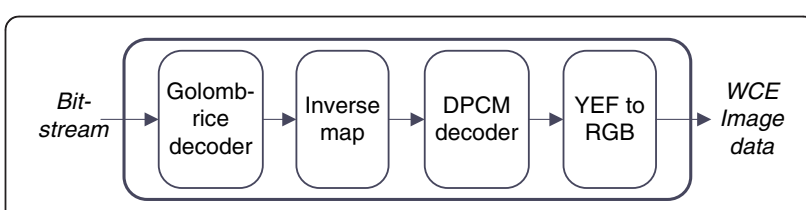

Figure 8 Block diagram of the image decoding algorithm. 


$$
\begin{aligned}
& \text { 1. } q:=\text { the number of } 0 \text { in the code until a } 1 \text { is reached } \\
& \text { 2. if } q<\left(g_{\text {limit }}-\log _{2} l-1\right) \text { then } \\
& \text { 3. } \quad r:=\text { next } k \text { bits in binary } \\
& \text { 4. } \quad m_{-} d x:=q \times M+r \\
& \text { 5. else } \\
& \text { 6. } \quad m \_d x:=\text { next } 8 \text { bits in binary }
\end{aligned}
$$

Figure 9 Pseudo code for Golomb-rice decoder.

several sub-sampling schemes (such as YEF812) on the chrominance (E and $\mathrm{F}$ ) components are applied. YEF812 sub-sampling means $\mathrm{Y}$ is not sub-sampled, $\mathrm{E}$ is subsampled after every 8 pixels, and $\mathrm{V}$ is sub-sampled after every 4 pixels. The sub-sampling is performed in horizontal direction only. A customized corner clipping scheme is also implemented to remove uninteresting corner area of the image to increase CR [33]. The proposed algorithm works in raster scan fashion and can directly be interfaced with commercial image sensors, eliminating the need of buffer memory. The compressor has an average CR of $80.4 \%$ and reconstructed image quality have peak signal-to-noise ratio (PSNR) index of about $43 \mathrm{~dB}$.

In the data logger, the decoder for the above discussed compression algorithm is implemented for RTV of images. The overall image decoding algorithm is shown in Figure 8.

In the compressed bit stream, the first pixel component is stored in raw 8-bit format. This is read in $X$, where $X$ is the actual pixel value. In YEF color space, $X$ corresponds to $Y, E$, and $F$ components. $X p$ is the next predicted pixel value. Initially

$$
X p=X
$$

Next we define

$$
\begin{aligned}
& I=2^{8}=256 \\
& M=2^{k},
\end{aligned}
$$

where $k$ is Golomb-rice code parameter. The maximum length of Golomb-rice code $\left(g_{\text {limit }}\right)$ is chosen as 32 . The pseudo code for Golomb-rice code decoder is shown in Figure 9.

The decoded $m \_d x$ is then inversely mapped to signed integer as shown in the pseudo code in Figure 10.

The differential pulse coded modulation decoder then calculates the next actual pixel value using (5) and sets the prediction for next pixel using (2).

$$
X=X p+d X
$$

After the actual component values of a pixel are calculated, they are converted to RGB color space using (6).

$$
\left[\begin{array}{l}
R \\
G \\
B
\end{array}\right]=\left[\begin{array}{ccc}
1 & 3.33 & 2.67 \\
1 & -2 & 0 \\
1 & 0.67 & -2.67
\end{array}\right]\left[\begin{array}{c}
Y \\
E-128 \\
F-128
\end{array}\right]
$$

The $R, G, B$ pixels are sent to graphical LCD for displaying the image frame. In order to display VGA images in QVGA size show, the VGA image is subsampled by 4. In Figure 11, the original and reconstructed images of an endoscopic image are shown.

\section{Data uploading}

After data logging, data can be uploaded to PC or Smartphone using any of the following three methods: (1) by removing the SD cards from data logger, (2) using USB interface, and/or (3) using wireless Bluetooth link. When using USB or Bluetooth link, a file transfer protocol is implemented which sends at first the filename, then the file size, and then its data bytes. A PC software is developed to receive the data from the data logger by USB or by Bluetooth. After receiving compressed data, the PC software can decode and display the images as video at any given FPS.

$$
\begin{aligned}
& \text { 1. If } m \_d x \bmod 2=0 \text { then } \\
& \text { 2. } d x:=m_{-} d x / 2 \\
& \text { 3. else } d x:=-\left(\left(m_{-} d X+1\right) / 2\right) \\
& \text { 4. } \quad d x
\end{aligned}
$$

Figure 10 Pseudo code for inverse mapping. 


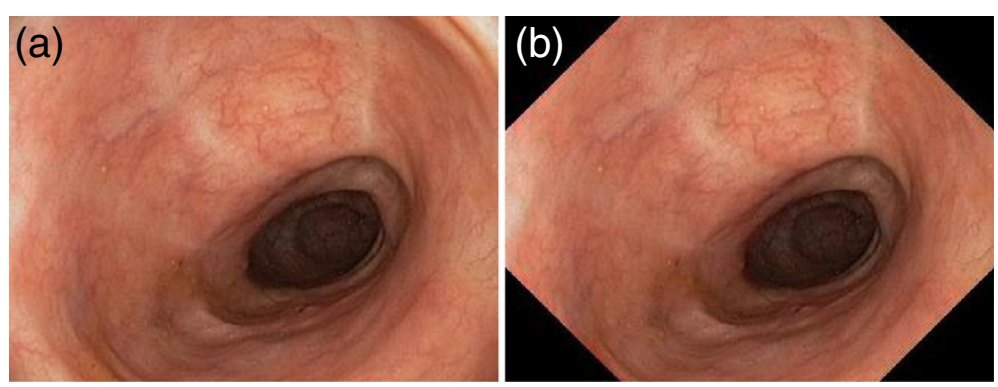

Figure 11 Comparison between original and reconstructed image (a) Original image. (b) Reconstructed image (PSNR is $43 \mathrm{~dB}$ ).
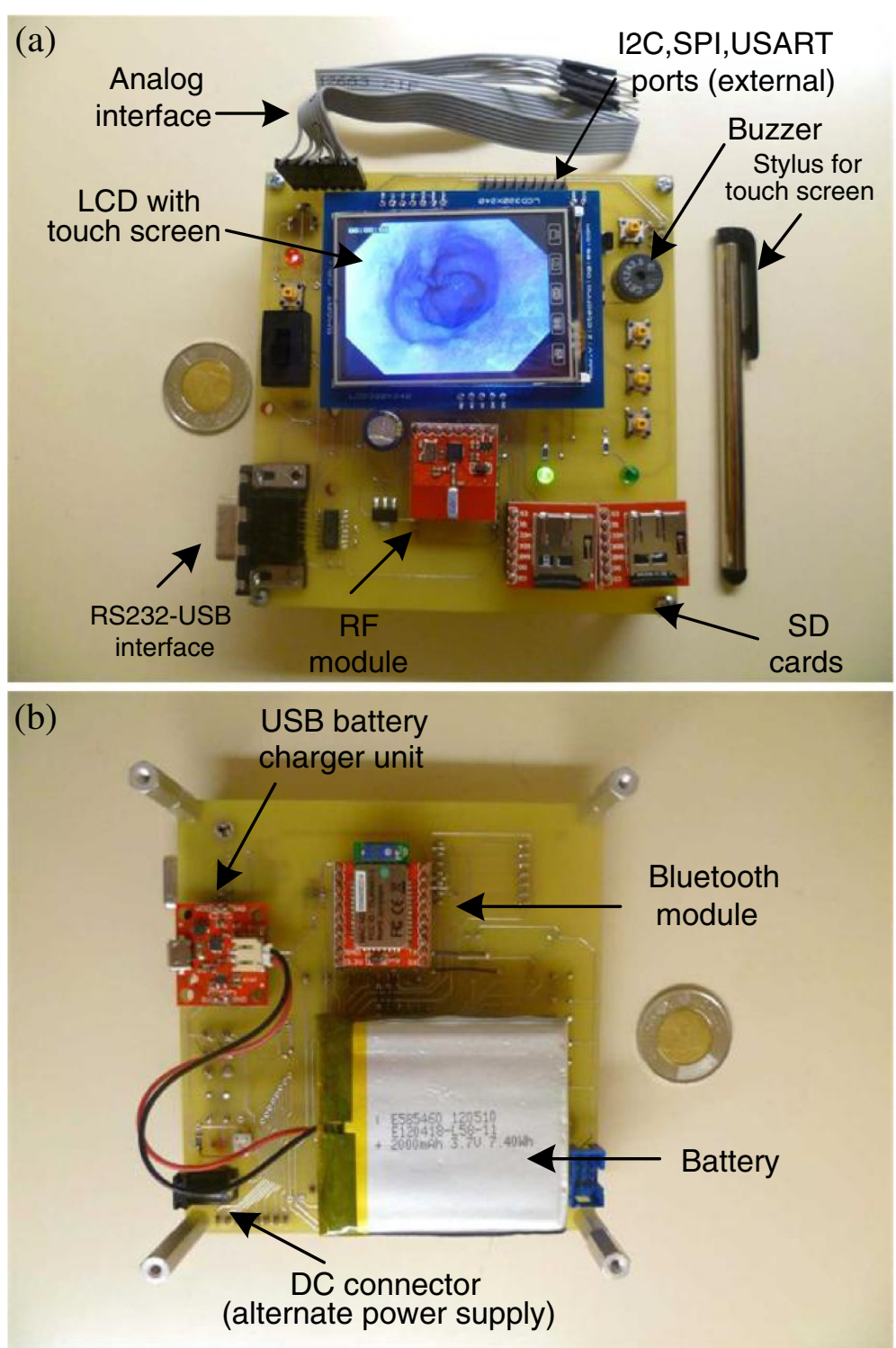

Figure 12 Photograph of the prototype. (a) Top view (compared with a Canadian \$2 coin). (b) Bottom view. 


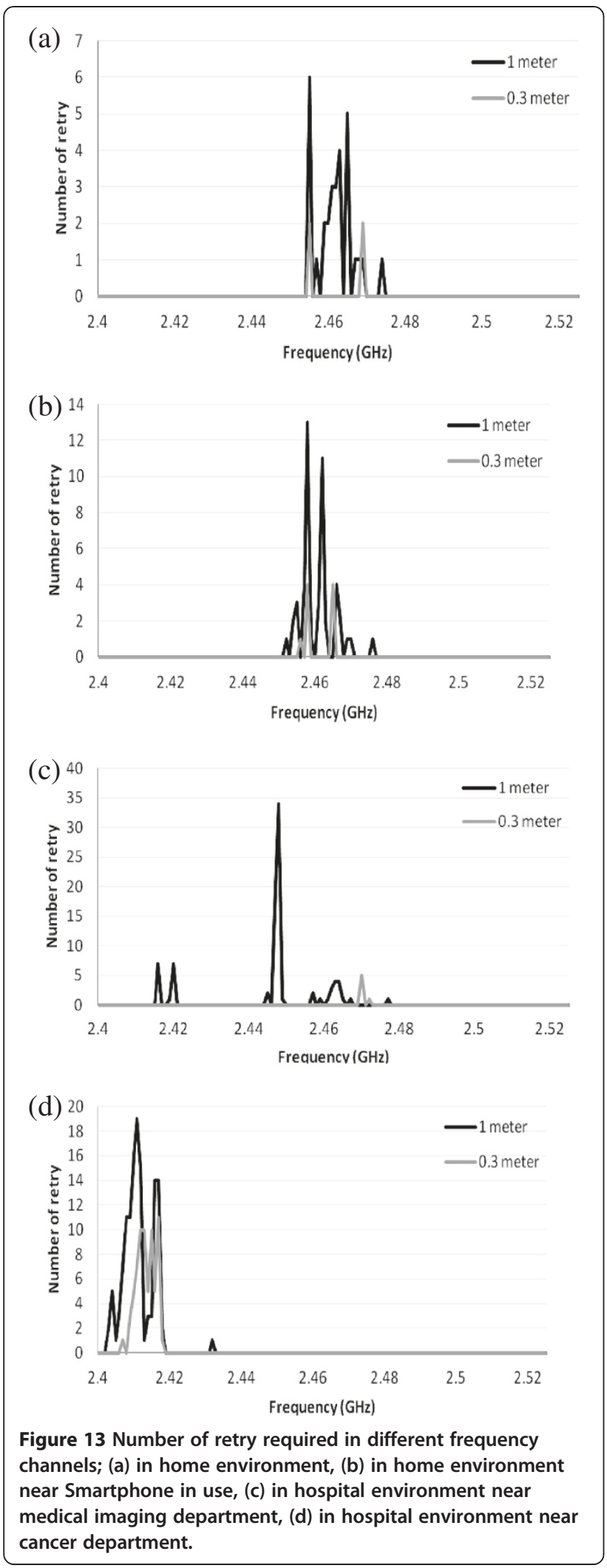

\section{Results}

\section{Specification of data logger prototype}

The proposed data logger for capsule endoscopy application is prototyped in our laboratory as shown in Figure 12 . The size of the prototype is $10 \times 10 \times 2 \mathrm{~cm}$. The weight of the PCB is $114 \mathrm{~g}$; each battery weighs $37 \mathrm{~g}$, making the total weight of the prototype $151 \mathrm{~g}$ with one battery. With three batteries, the total weight is $114+$ $(3 \times 37)=225 \mathrm{~g}$. Note that, for capsule endoscopy application, minimum 10-h battery life is required. If we use one battery instead of three batteries then the hardware can run for $35.3 / 3=11.8 \mathrm{~h}$, which meets the design requirement for capsule endoscopy application. As the design of the proposed data logger is general, three batteries are used to meet other applications which may require more data logging time.

\section{Experimental results for RF channel selection}

We have conducted experiments to observe the interference of other frequencies (such as Wi-Fi, Smartphone, medical instruments, etc.) in both hospital (Royal University Hospital, Saskatoon, SK, Canada) and home environment [15] on the Nordic transceiver in its different frequency channels. Several experiments showed that using duck antenna [34] instead of chip antenna improves the RF transmission quality. In Figure 13, the number of retry required by the transceiver to send 100 data packets (each packet contains 32 bytes) in different frequency channels in several environments using duck antenna is shown. As the data logger is wearable, the distance between the sensor and the logger should be less than $1 \mathrm{~m}$ (taking a conservative approach).

From Figure 13, we see that several retries are required from 2.4 to $2.48 \mathrm{GHz}$ channels due to interference in different environments. So, we have decided to operate the transceiver in a clear channel at $2.5 \mathrm{GHz}$ which will cause minimum number of retries during data transmission. Note that, the Bluetooth device will not have any interference effect on Nordic transceiver as they work in a mutually exclusive way.

It is possible to select the best channel dynamically during data logging. The procedure is as follows.

1. The data logger will go to channel selection mode after a configurable time (for instance, every after $30 \mathrm{~min}$ ) or by user at anytime from selecting a menu option.

2. The data logger will send a command to the biosensor to set its transceiver at the first channel and the data logger will also set its transceiver to the first channel. Then the data logger will send some datapackets to the bio-sensor and log the number of retry required for that channel. This step will be done for all the available channels (in Nordic 
transceiver, a total of 126 channels starting from 2.4 to $2.526 \mathrm{GHz}$ are available).

3. Then the channel which has minimum retries will be selected and the data logger will send a command containing the channel number to the bio-sensor so that both data logger and bio-sensor communicates at the same channel.

\section{Experimental results simulating capsule endoscopy scenario}

An MCU-based capsule emulator hardware as shown in Figure 14 is developed for testing the data logger prototype. The compression algorithm proposed in [32,33] is implemented in a PC software and compressed bitstream data for several endoscopic images are generated using the software. Then the compressed bit-stream data are downloaded in the program-memory of the MCU of the capsule emulator. When the capsule emulator receives a command from the data logger through the RF interface, it starts to send compressed image data to the data logger wirelessly.

In order to test the data logger for a capsule endoscopy scenario, the capsule emulator hardware is inserted inside a turkey and the data logger is placed 0.3-m away from the capsule as shown in Figure 15. The data logger received images wirelessly through the turkey's skin and flesh successfully from the capsule. As the data logger is wearable and it is generally worn at one side of the belly, the distance between a swallowed capsule and data logger will be near $0.3-\mathrm{m}$ for human endoscopy. The data logger is also placed about $1 \mathrm{~m}$ apart from the inserted capsule and images transmitted successfully.

\section{Experiment results for multiple body sensors}

In order to test the wired analog interfaces of the data logger prototype, two temperature sensors [35] are connected with two analog ADC channels. The temperature sensor gives analog voltage output proportional to its surface temperature $\left(0.01 \mathrm{~V} /{ }^{\circ} \mathrm{C}\right)$. The sensors are placed on two places of a human hand as shown in Figure 16. The data logger is programmed to sample data at $10 \mathrm{~Hz}$. It converts the sampled analog data to digital, stores them in SD card along with time stamp,

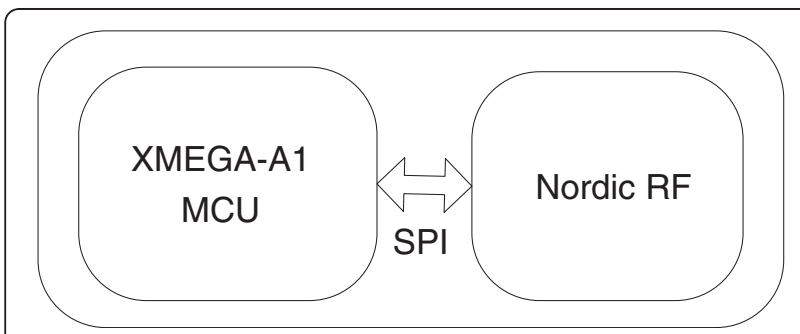

Figure 14 Block diagram of capsule emulator.

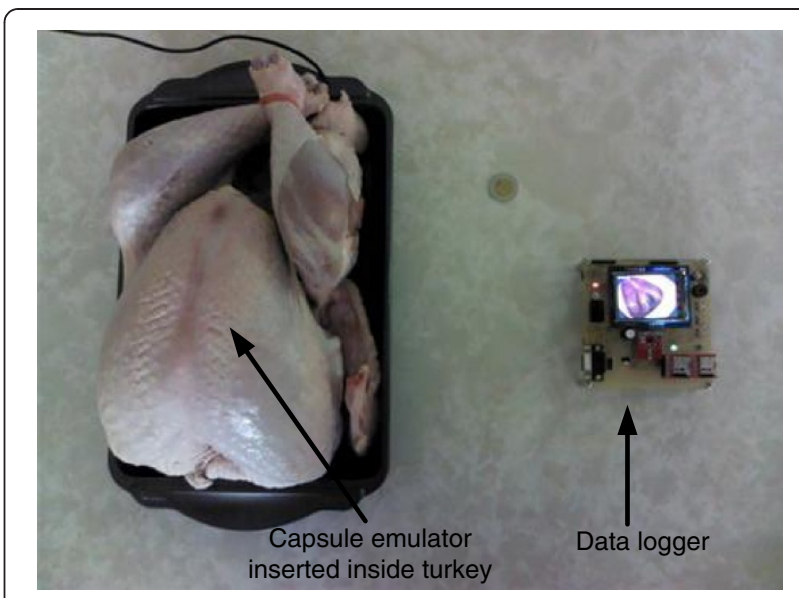

Figure 15 Experimental setup: capsule emulator is placed inside a turkey and the data logger is placed outside.

and plots the data on graphical LCD for real-time display as shown in Figure 16. The logged data can be transferred to $\mathrm{PC}$ or Smartphone and further analysis can be done. By using the analog channels, blood pressure, ECG, heartbeat signals can also be logged and displayed.

\section{Power consumption}

The power consumption of the data logger in different modes is shown in Table 4. When RTV is disabled, the graphical LCD is taken to sleep mode to save power. In Figure 17, the percentage power consumption of different hardware components during data logging (using Nordic RF link, in RTV enabled mode) is shown. We see that the LCD consumes the majority of power (54\%) if the RTV is enabled. It should be noted that the RTV mode is not a normal mode of operation, rather a special feature that a physician may use if desired. After the data logging is completed, it can be transferred to PC by removing the SD card, which costs no power for the data logger. When transferring data using Bluetooth, the LCD and Nordic RF are taken to sleep mode to save power. During data transfer by USB interface, the Bluetooth is also taken to sleep mode.

\section{Comparison with other works}

The overall specification of the designed data logger and its comparison with other works are shown in Table 5. Comparing with other works on data loggers in the literature, the proposed data logger has high-speed wireless connectivity including Bluetooth, contains graphical LCD for real-time data viewing, and touch screen. The storage space is sufficient for many other medical applications; moreover, it can be increased easily by connecting more SD cards as shown in Figure 4 or implementing FAT32 file system in the firmware which 

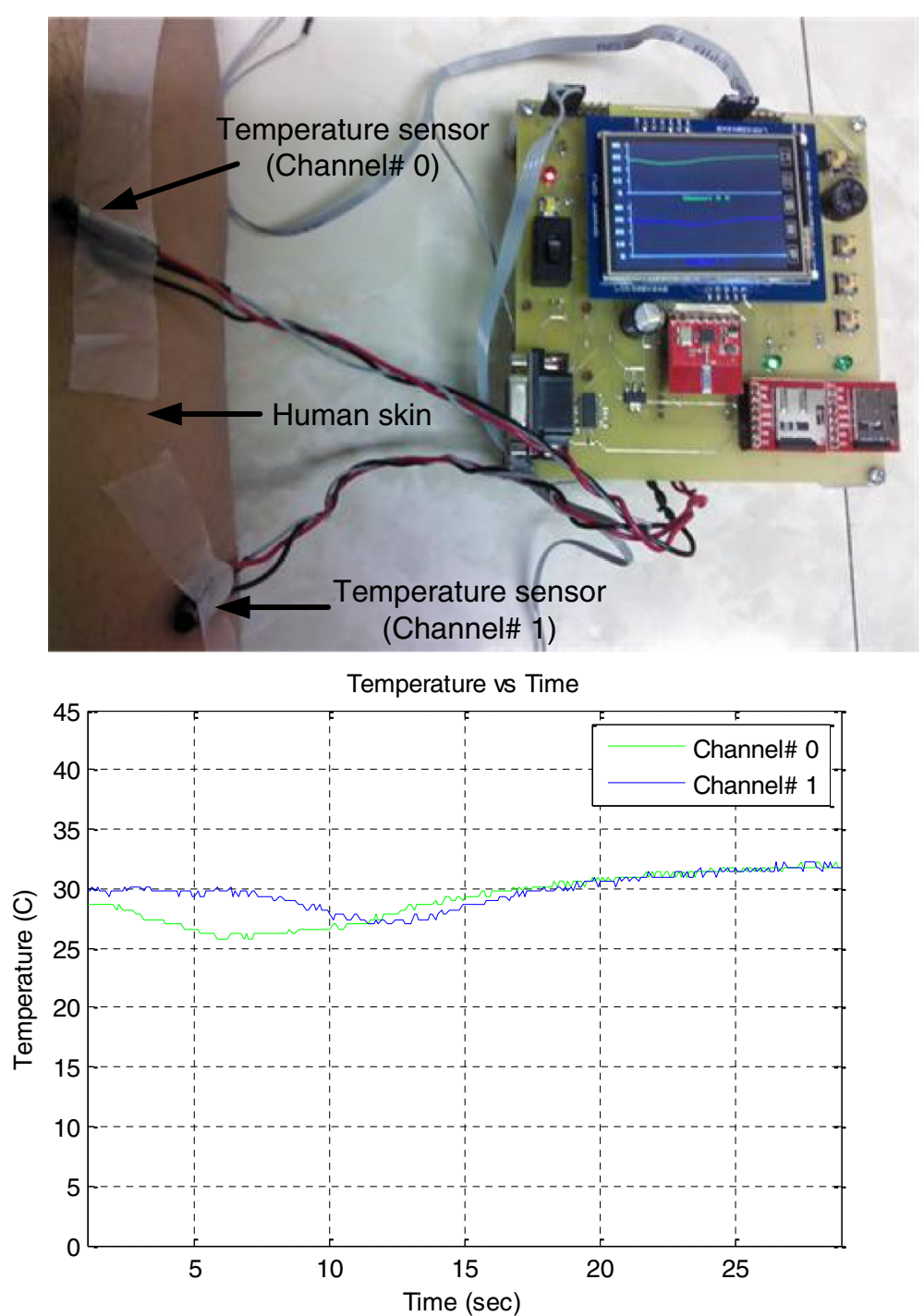

Figure 16 Skin temperature logging and display: (a) real-time display on LCD; (b) logged data plotted in MATLAB.

Table 4 Power consumption in different modes

\begin{tabular}{lll}
\hline Mode & Current $(\mathbf{m A})$ & Power $(\mathbf{m W})$ @ 3.3 V \\
\hline Display menu & 160 & 528 \\
Data logging in SD card & & \\
RTV enabled & 170 & 561 \\
RTV disabled & 90 & 297 \\
Data transfer to PC & & \\
Using SD card reader & 0 & 0 \\
Bluetooth & 140 & 462 \\
USB interface & 93 & 307 \\
\hline
\end{tabular}

can support higher capacity SD card (8 GB or higher) than FAT16. The writing speed and RF bandwidth of the data logger is sufficiently high to capture and store data at high sampling rate. Thus, the proposed data logger fulfills all the design requirements as discussed in Design requirements section for capsule endoscopy application. The data logger is designed for capturing images and images need more RF bandwidth, disk space, writing speed, etc., than recording other physiological signals such as heart beat, blood pressure, temperature, etc. So, the proposed data logger can be used efficiently in other medical applications.

\section{Discussion}

\section{Wireless data transmission}

The medical implantable communication service (MICS) compatible RF transceiver, which works at 402-405 


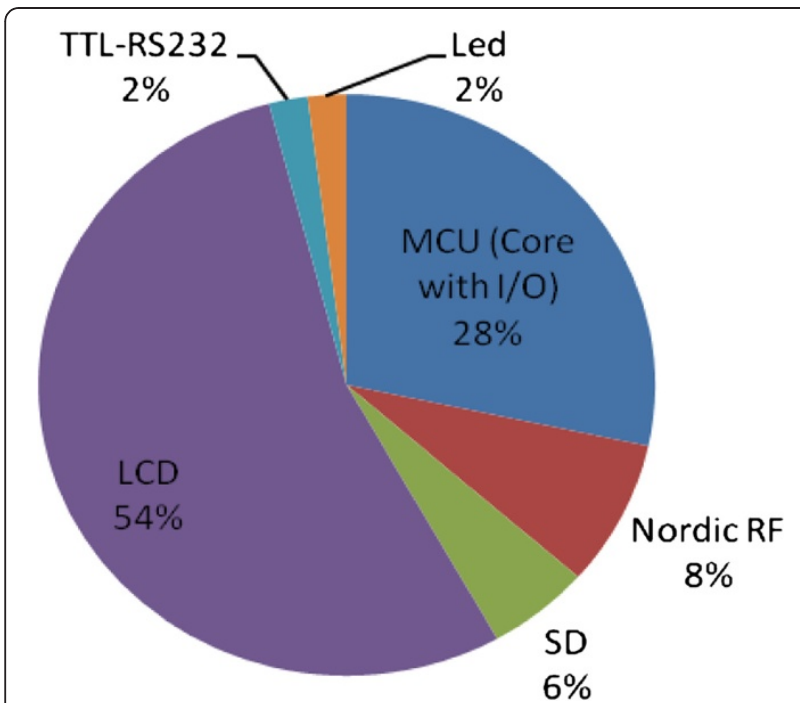

Figure 17 Percentage power consumption of different hardware components.

$\mathrm{MHz}$ frequency, is the most suitable for transmitting data through the human body [36]. However, the MICS RF transceiver vendor, Zarlink [24], sells a low data rate (effective data rate around $500 \mathrm{kpbs}$ [37]) transceiver (ZL70102) which is not sufficient even for 2 FPS capsule endoscopy application. Zarlink has a custom transceiver (ZL70081) having a data rate of $2.7 \mathrm{Mbps}$ which is not available for public purchase. So, we used the RF transceiver by Nordic [23], which works at $2.4 \mathrm{GHz}$ frequency band and having a data rate of 2 Mbps. However, the effective data rate with minimum header information is 1.68 Mbps. The studies in $[37,38]$ show that $2.4 \mathrm{GHz}$ transceivers, such as Nordic, can effectively be used to get data wirelessly through animal body.

In this design, the Nordic transceiver was configured in auto acknowledgement mode. In auto acknowledge mode, after receiving a data packet, the receiver checks the CRC bits and detects whether there was any error during the transmission of the packet. If there was any error, then it requests the transmitter to resend the data
Table 6 Engineering cost of the data logger

\begin{tabular}{lccc}
\hline Task & $\begin{array}{c}\text { Time } \\
\text { required (hours) }\end{array}$ & $\begin{array}{c}\text { Personnel } \\
\text { qualification }\end{array}$ & $\begin{array}{c}\text { Number of } \\
\text { personnel }\end{array}$ \\
\hline $\begin{array}{l}\text { Requirement } \\
\text { analysis }\end{array}$ & 40 & $\begin{array}{c}\text { Researcher } \\
\text { (graduate } \\
\text { student) }\end{array}$ & One \\
$\begin{array}{l}\text { Specification and } \\
\text { system design }\end{array}$ & 24 & Embedded & One \\
$\begin{array}{l}\text { Hardware/software } \\
\text { partitioning }\end{array}$ & 16 & $\begin{array}{c}\text { Estem engineer } \\
\text { (or similar) }\end{array}$ & \\
$\begin{array}{l}\text { Purchasing } \\
\text { components, tools, } \\
\text { and compilers }\end{array}$ & 5 & & \\
$\begin{array}{l}\text { Hardware design } \\
\text { Firmware design }\end{array}$ & 40 & & \\
$\begin{array}{l}\text { Prototyping, } \\
\text { debugging, and } \\
\text { testing }\end{array}$ & 40 & & One \\
$\begin{array}{l}\text { PCB design and } \\
\text { testing }\end{array}$ & 40 & & \\
\hline
\end{tabular}

packet again. This process goes on until the packet is transmitted successfully. So, in auto acknowledgement mode, no data loss happens, though the number of retries will decrease the overall data rate or application throughput. If the receiver is unable to detect error using $\mathrm{CRC}$, then depending upon which bits get corrupted, the reconstructed image will have different distortions. The image compression algorithm [32,33] used here is linebased differential coding (i.e., works row-by-row in raster scan fashion). In some cases, one row (or part of a row) of the image pixels may get corrupted if undetectable errors occurs in data packets. In worst case, the entire image frame may get corrupted.

Commercial capsule endoscopy products [6] such as PillCam SB, EndoCapsule, and MiroCam send images of size $256 \times 256,256 \times 256$, and $320 \times 320$, respectively. VGA $(640 \times 480)$ images are difficult to sent at 2 FPS because it requires very high data rate custom design RF transmitter. Though, some research works are available in the literature $[39,40]$ on high data rate (as high as 15

Table 5 Comparison with other data loggers

\begin{tabular}{|c|c|c|c|c|c|c|c|}
\hline & $\begin{array}{l}\text { Memory } \\
\text { capacity }\end{array}$ & $\begin{array}{l}\text { Sampling rate } \\
\text { (writings speed) }\end{array}$ & Wireless & Analog interface & Graphical LCD & Touch input & Battery life (hour) \\
\hline [7] & $16 \mathrm{MB}$ & $13.2 \mathrm{kHz}$ & No & Yes & No & No & 50 \\
\hline [2] & $20 \mathrm{MB}$ & $200 \mathrm{~Hz}$ & No & Yes & No & No & - \\
\hline [8] & - & $500 \mathrm{~Hz}$ & No & Yes & No & No & 24 \\
\hline [15] & - & $60 \mathrm{~Hz}$ & $250 \mathrm{kbps}$ & No & No & No & $-{ }^{\mathrm{a}}$ \\
\hline [3] & $512 \mathrm{MB}$ & $33 \mathrm{kS} / \mathrm{s}$ & No & Yes & No & No & 72 \\
\hline$[30]$ & $512 \mathrm{kB}$ & - & No & Yes & No & No & 0.23 \\
\hline [11] & $512 \mathrm{MB}$ & - & No & Yes & No & No & 93.6 \\
\hline This study & $4 \mathrm{~GB}^{\mathrm{b}}$ & 192 k pixel/s ${ }^{c}$ (115 kbps) & 2 Mbps link and Bluetooth & Yes & Yes & Yes & 35.3 \\
\hline
\end{tabular}

${ }^{a}$ not battery operated, ${ }^{b}$ expandable, ${ }^{\text {f }}$ for $80 \% \mathrm{CR}$. 
Table 7 Manufacturing cost of the data logger

\begin{tabular}{ll}
\hline Item & Price (USD) \\
\hline Hardware components & $\$ 180$ \\
(LCD, MCU, RF unit, Bluetooth unit, misc) & \\
Programmer unit (one-time cost) & $\$ 40$ \\
PCB fabrication & $\$ 40$ \\
Total & $\$ 260.00$ \\
\hline
\end{tabular}

Mbps) transmitter for capsule endoscopy, however, they are not commercially available yet. So, the bottleneck of sending VGA images at 2 FPS is the RF transmitter. However, VGA images can be sent at lower FPS using the limited available bandwidth of the commercially available RF transmitters. In order to send VGA color images at $1 \mathrm{FPS}$, having $80 \% \mathrm{CR}$ for $10 \mathrm{~h}$, the memory space, the RF data rate, and the writing speed should be at least $6.2 \mathrm{~GB}, 1440 \mathrm{kbps}$, and $180 \mathrm{kB} / \mathrm{s}$, respectively. To increase the size of memory capacity, the number of SD cards as shown in Figure 4 can be increased to more than 2. The MISO, MOSI, and SCK pins of the additional SD cards will be connected directly with the MCU's MISO, MOSI, and SCK pins and extra SS pin from the MCU needs to connect with the additional SD cards SS pins. Moreover, memory capacity can be also increased without changing any hardware design. Secure Digital High Capacity (SDHC) cards can be used instead of SD cards which have a capacity of $32 \mathrm{~GB}$ and have higher writing speed than SD cards [41]. In order to read/write data in SDHC, FAT32 file system needs to be implemented in the firmware instead of FAT16.

\section{Cost of developing the data logger prototype}

The engineering cost of the proposed data logger is approximately around $260 \mathrm{~h}$. The task list, tentative time required for each task, qualification, and number of personnel are shown in Table 6. This approach can easily be adapted by other researchers interested in similar development. The manufacturing cost of the data logger is shown in Table 7. It should be noted that the cost of programmer module is one-time. For bulk manufacturing, the cost will significantly be lower.

\section{Future works}

A small size FPGA-based capsule prototype is now under development where a novel image compression algorithm proposed in $[32,33]$ is implemented. After the final capsule prototype is developed, it will be inserted in pigs intestine [42] due to its relatively similar gastrointestinal functions in comparison to human and live images will be captured for more rigorous animal testing.

Testing the data logger with capsule endoscopy prototype in several human patients faces several challenges. A number of capsule prototypes need to be manufactured which requires significant amount of time, money, and human resource. Then the permission from Food and Drug Administration [43] needs to be taken for swallowing the prototype capsules in human. Moreover, it requires approval from hospital, managing a number of patients having gastrointestinal diseases, and involvement of gastroenterologist to evaluate the performance [44] and measuring the accuracy of their decision (fraction of correct decisions, false positives, false negatives, etc.) using statistical tools such as receiver operating characteristics analysis [45]. These tasks are beyond the scope of the article and left for future exploration.

\section{Conclusion}

In this article, the design of a microcontroller-based portable data logger is presented that is targeted for general medical imaging and data collection application. It has high data rate wireless connectivity including Bluetooth, graphical display for real-time data viewing with state-of -the-art touch screen technology. The data are logged in micro SD cards and can be transferred to PC or Smartphone using card reader, USB interface, or Bluetooth wireless link. The features of the prototype are demonstrated for a WCE and skin temperature logging application.

\section{Competing interests}

The authors declare that they have no competing interests.

\section{Acknowledgments}

This study was supported by the Natural Science and Engineering Research Council of Canada (NSERC) and the Canada Foundation for Innovation (CFI). The authors would like to give thanks to Mohammad Shamim Imtiaz for helping in collecting data from hospital environment and to Serge Nazerenko for his technical assistance in making the prototype.

Received: 8 August 2012 Accepted: 20 November 2012 Published: 26 December 2012

\section{References}

1. P. Corbishley, E. Rodriguez-Villegas, Breathing detection: towards a miniaturized, wearable, battery-operated monitoring system. IEEE Trans. Biomed. Eng. 55(1), 196-204 (2008). doi:10.1109/TBME.2007.910679

2. G. Cybulski, A. Ksiazkiewicz, W. Lukasik, W. Niewiadomski, T. Palko, Ambulatory monitoring device for central hemodynamic and ECG signal recording on PCMCl flash memory cards. Comput. Cardiol. 1995, 505-507 (1995). doi:10.1109/CIC.1995.482712

3. R. Rieger, Y.R. Huang, A custom-design data logger core for physiological signal recording. IEEE Trans. Instrum. Meas. 60(2), 532-538 (2011). doi:10.1109/TIM.2010.2051609

4. T. Deniz, A. Yilmaz, Design and implementation of a digital ambulatory ECG recorder based on flash MultiMediaCard memory, in Proceedings of the IEEE 46th Midwest Symposium on Circuits and Systems, 2003, vol. 1, pp. 368-371. doi:10.109/MWSCAS.2003.1562295. Cairo

5. U. Anliker, J. Ward, P. Lukowicz, G. Tröster, F. Dolveck, M. Baer, F. Keita, E. Schenker, F. Catarsi, L. Coluccini, A. Belardinelli, D. Shklarski, M. Alon, E. Hirt, R. Schmid, M. Vuskovic, AMON: A wearable multi parameter medical monitoring and alert system. IEEE Trans. Inf. Technol. Biomed. 8(4), 415-427 (2004). doi:10.1109/TITB.2004.837888

6. J.L. Toennies, G. Tortora, M. Simi, P. Valdastri, R.J. Webster, Swallowable medical devices for diagnosis and surgery: the state of the art. Proc. IMechE C: J. Mech. Eng. Sci. 224, 1397-1414 (2009). doi:10.1243/09544062JMES1879

7. R. Luharuka, R.X. Gao, S. Krishnamurty, Design and realization of a portable data logger for physiological sensing [GSR]. IEEE Trans. Instrum. Meas. 52(4), 1289-1295 (2003). doi:10.1109/TIM.2003.816808 
8. F. Ahmed, M.A. Mohd Ali, E. Zahedi, Development of a portable fetal and maternal heart rate recorder for 24 hours, in IEEE Proceedings of the 22 nd Annual International Conference on Engineering in Medicine and Biology Society, 2000, vol. 4, pp. 3044-3047. doi:10.1109/IEMBS.2000.901523. Chicago, IL

9. A. Salarian, H. Russmann, F.J.G. Vingerhoets, P.R. Burkhard, K. Aminian, Ambulatory monitoring of physical activities in patients with Parkinson's disease. IEEE Trans. Biomed. Eng. 54(12), 2296-2299 (2007). doi:10.1109/ TBME.2007.896591

10. B. Hermans, R. Puers, A portable multi-sensor data-logger for medical surveillance in harsh environments. Sens. Actuators A: Physical (Elsevier) 123-124, 423-429 (2005)

11. F.S. Jaw, Y.L. Tseng, J.K. Jang, Modular design of a long-term portable recorder for physiological signals. Measurement (Elsevier) 43(10), 1363-1368 (2010)

12. E. Ryo, K. Nishihara, S. Matsumoto, H. Kamata, A new method for long-term home monitoring of fetal movement by pregnant women themselves. Med. Eng. Phys. (Elsevier) 34(5), 566-572 (2012)

13. R.K. Kamat, S.A. Shinde, P.K. Gaikwad, H. Guhilot, Analog front end and FPGA based soft IP core for ECG logger, in Harnessing VLSI System Design with EDA Tools, (Springer), 2012, pp. 51-91. doi:10.1007/978-94-007-1864-7_3

14. C. Tronstad, S. Grimnes, O.G. Martinsen, E. Fosse, Development of a medical device for long-term sweat activity measurements, in Proceedings of the IFMBE. vol. 17 (Springer, Berlin, 2007), pp. 236-239. Graz, Austria

15. C. Chao, C. Pomalaza-Raez, Design and evaluation of a wireless body sensor system for smart home health monitoring, in Proceedings of the IEEE Global Telecommunications Conference, 2009, pp. 1-6. doi:10.1109/ GLOCOM.2009.5425471. Honolulu, HI

16. P. Augustyniak, Wearable wireless heart rate monitor for continuous longterm variability studies. J. Electrocardiol. (Elsevier) 44(2), 195-200 (2011)

17. A. Takeuchi, N. Mamorita, F. Sakai, N. Ikeda, Development of a comprehensive medical recorder on a cellphone. Comput. Methods Programs Biomed. (Elsevier) 97(1), 28-38 (2010)

18. Atmel Corporation, 2012. [Online], http://www.atmel.com/products/ microcontrollers/avr/default.aspx

19. Microchip Technology Inc, PIC16F1947 microcontroller, 2012. http://www. microchip.com/wwwproducts/Devices.aspx?dDocName=en538146

20. NXP Semiconductors, 32 bit ARM Cortex-M3 microcontroller, 2012. http:// www.nxp.com/products/microcontrollers/cortex_m3/LPC1316FBD48. html\#overview

21. Smart GPU, Vizic technologies, 2012. http://vizictechnologies.com/\#/smartgpu/4554296549

22. SanDisk microSD, 2012. http://www.sandisk.com/products/memory-cards/ microsd/

23. Nordic nRF24L01+ transceiver, 2012. http://www.nordicsemi.com/eng/ Products/2.4GHz-RF/nRF24L01P

24. Zarlink Semiconductor, ZL70102 MICS transceiver, 2012. http://www.zarlink. com/zarlink/hs/82_ZLE70102.htm

25. Linx Technologies, LR series transmitter, TXM-433-LR, 2012. http://www. linxtechnologies.com/resources/data-guides/txm-xxx-Ir.pdf

26. QwikRadio ${ }^{\text {TM }}$ UHF ASK Transmitter, MICRF103, 2012. http://www. datasheetcatalog.org/datasheet/Micrel/mXsvwwt.pdf

27. XBee RF modules, 2012. http://www.digi.com/products/wireless-wiredembedded-solutions/zigbee-rf-modules/point-multipoint-rfmodules/xbeeseries1-module\#overview

28. Roving Networks, Class 1 Bluetooth Module, RN-41, 2012. http://www. rovingnetworks.com/products/RN_41

29. Texas Instruments, TPS61200 boost converter, 2012. http://www.ti.com/ product/tps61200

30. Microchip Technology Inc, MCP73831/2 Linear charge management controller, 2012. http://www.microchip.com/wwwproducts/Devices.aspx? dDocName $=$ en024903

31. MCS Electronics, AVR-DOS, 2012. http://www.mcselec.com/index.php? page $=$ shop.product_details\&flypage $=$ shop. flypage\&product_id=31\&category_id=6\&option=com_phpshop\&ltemid=1

32. T.H. Khan, K. Wahid, Low complexity color-space for capsule endoscopy image compression. IET Electron. Lett. 47(22), 1217-1218 (2011). doi:10.1049/el.2011.2211

33. T.H. Khan, K. Wahid, Low power and low complexity compressor for video capsule endoscopy. IEEE Trans. Circuits Syst. Video Technol. 21(10), 15341546 (2011). doi:10.1109/TCSVT.2011.2163985
34. Chang Hong Technology Co., Ltd, 2.4GHz dipole antenna, 2012. http://www. sparkfun.com/datasheets/Wireless/Antenna/DA-24-04.pdf

35. Texas Instruments, LM35 Precision Centigrade Temperature Sensor, 2012 http://www.ti.com/lit/ds/symlink/lm35.pdf

36. FCC rules and regulations 47 CFR Part 95, subparts E (95.601-95.673) and I (95.1201-95.1219) Personal Radio Services, 2002

37. A.R. Kahn, E.Y. Chow, O.A. Latief, P.P. Irazoqui, Low-power, high data rate transceiver system for implantable prostheses. Int. J. Telemed. Appl., Article ID 563903 (2010). doi:10.1155/2010/563903

38. P. Valdastri, A. Menciassi, P. Dario, Transmission power requirements for novel Zigbee implants in the gastrointestinal tract. IEEE Trans. Biomed. Eng. 55(6), 1705-1710 (2008)

39. S. Diao, Y. Zheng, Y. Gao, C.H. Heng, M. Je, A 7.2mW 15Mbps ASK CMOS transmitter for ingestible capsule endoscopy, in Proceedings of the IEEE Asia Pacific Conference on Circuits and Systems (APCCAS), 2010, pp. 512-515. Kuala Lumpur

40. S. Stoa, R.C. Santiago, I. Balasingham, An ultra wideband communication channel model for capsule endoscopy, in Proceedings of the International Symposium on Applied Sciences in Biomedical and Communication Technologies (ISABEL), 2010, pp. 1-5. Rome

41. SD Card vs SDHC, Diffen [Online], 2012. Available at http://www.diffen.com/ difference/SD_Card_vs_SDHC

42. M. Kopáčová, I. Tachecí, J. Květina, J. Bureš, M. Kuneš, S. Spelda, V. Tyčová, Z. Svoboda, S. Rejchrt, Wireless video capsule enteroscopy in preclinical studies: methodical design of its applicability in experimental pigs. Dig. Dis. Sci. 55(3), 626-630 (2010). doi:10.1007/s10620-009-0779-3

43. Food and Drug Administration, 2012. http://www.fda.gov

44. D. Tokuhara, K. Watanabe, Y. Okano, A. Tada, K. Yamato, T. Mochizuki, J. Takaya, T. Yamano, T. Arakawa, Wireless capsule endoscopy in pediatric patients: the first series from Japan. J. Gastroenterol. (Springer) 45, 683-691 (2010)

45. T. Fawcett, An introduction to ROC analysis. Pattern Recognit. Lett. 27, 861-874 (2006)

doi:10.1186/1687-3963-2012-10

Cite this article as: Khan and Wahid: An advanced physiological data logger for medical imaging applications. EURASIP Journal on Embedded Systems 2012 2012:10.

\section{Submit your manuscript to a SpringerOpen ${ }^{\odot}$ journal and benefit from:}

- Convenient online submission

- Rigorous peer review

- Immediate publication on acceptance

- Open access: articles freely available online

- High visibility within the field

- Retaining the copyright to your article

Submit your next manuscript at $>$ springeropen.com 